\title{
Equilibrium Determination, Two-Sided Markets And Platforms Competition: The Nigerian Mobile Communication Market
}

\author{
Prof Francis A. Akawu \\ Department of Economics, \\ Nasarawa State University, Keffi, Nigeria. \\ Atsiya Pius Amos \\ Department of Economics and Related Studies, \\ University of York, United Kingdom. \\ Department of Economics, \\ Nasarawa State University, Keffi, Nigeria.
}

\begin{abstract}
This study develops a theoretical model for analyzing two-sided markets that allow for global multi-homing among agents in a mobile communication market. The model explains the determination of equilibrium outcome, price structure and consumer surplus given competitive platforms and non-exclusivity of services in the Nigerian Mobile communication market. Multiple equilibria, as well as maximum platform profit from monopoly rent with associated deadweight losses on the side of the buyers, are feasible given the market structure. The existing pricing structure is seemingly suboptimal of a social welfare. The unique characteristic of the market having mixed features of monopoly and perfect competition appears to be critical in reaching the theoretical model outcome. Consumer welfare is likely to be promoted with strict enforcement of anti-trust policy needed to regulate any form of collusion.
\end{abstract}

Keywords: Two-sided markets, multi-homing, platform competition

JEL Classification: L15, 96

\section{INTRODUCTION}

Technological advancement has resulted in the growth of markets that operate via platforms in developing countries. Such platforms serve as intermediaries that connect different groups of agents in a market. The two-sided market is typical of the mobile communication industry where two distinct sets of agents interact via platforms. Another relevant example are dating sites (platforms) were match-making are executed. Choice of action by all categories of players is crucial in the determination of the efficiency of the market. The decision of agents to singlehomes (operating through one platform) or multi-home (using several platforms) could affect significantly, their expected gains (Armstrong, 2006). Similarly, the platform efficiency in matching agents is critical in determining agents' welfare. In Nigeria, the Public Switch Telephone Network (PSTN) is the gateway mobile switching platform providing connectivity among network operators. It also provides connectivity between mobile phone users and software developers (agent-types) in the Nigerian mobile communication market. Nitel and Globacom are the two PSTNs in Nigeria . Following Armstrong (2006), three alternatives relations are available to these groups (mobile phone users and software developers) of agents: (i) both groups can decide to single home (ii) one group single home (mobile phone users) while the other group multi-homes (software developers) (iii) both groups decide to multi-homes. The last alternative defines the study market. 
The Nigeria mobile communication market witnessed a major revolution following the Digital Mobile License (DML) issued to Global System for Mobile (GSM) telecommunication providers such as AIRTEL, MTN, and MTEL in 2001, GLOBACOM in 2002 and ETISALAT in 2007. This has resulted in a phenomenal growth in the Nigerian teledensity from 16.7 percent in 2001 to 91.7 percent in 2015 (NTS-NBS, 2015). A similar increase in the penetration connections from 54 percent in 2010 to 80 percent in 2014 has been witnessed (GSMA Intelligence, 2014). At present, the Nigerian Telecommunications Ecosystem comprises of five major Mobile Network Operators (HMM, GLO, AIRTEL, 9MOBILE, NTEL and MTN), four Internet Service Providers (SMILE, IPNX, SWIFT, SPECTRANET) and major Technology Services Companies such as SEAMFIX, HUAWEI, MICROSOFT, TECHNOTREE, CISCO, TECHMAHINDRA. The Original Equipment Manufacturer/Phone Manufacturers which include ERICSON, HUAWEI, MICROSOFT, and CISCO provide services such as: Mobile Financial Services, Mobile Advertising Services, Mobile Health Services, Lumos, and Mobile Electricity. In the face of a seemingly "robust" telecommunication ecosystem, the efficiencies of service providers appear to be in doubt. Based on a survey report, the mean customer satisfaction index score (a proxy for quality of service) was 59.1 percent with the extent to which services meet expectations as the most poorly rated in Africa (NCS, Report, 2012).

Essentially, the study is aimed at the determination of equilibrium outcome and its desirable properties in a market that exhibits features of both perfect competition and monopoly. The study is motivated by several observations about two-sided mobile communication market in Nigeria. The specificity of these features in relation to the market performance stimulated the development of a model to explain the activities of the market. The simple model developed is expected to explain how equilibrium outcome, price structure, and consumer surplus are determined. At present, prices in the market are endogenously determined and this is inconsistent with the Ramsey policy but permits the attainment of maximum profit at equilibrium through the instrument of the transaction fee. The capability of agents to extract their surpluses from trade appears to differ with mobile phone users. Resorting to price regulation as suggested by Rochet and Tirole (2002) would require an explanation on how sub-markets should operate to elicit the desired outcome.

The market is characterized by several pricing problems. First is the Ramsey problem where prices are not reflective of the marginal cost and are usually above the social optimal. Second is the arbitrary determination of prices and the practice of using the surplus profit generated from a market side with smaller elasticity to subsidize the other side with greater elasticity. Third, the presence of third-degree price discrimination on one side of the market. Pricing by intermediaries has generated concerns on the margin between gross utility and transaction fee -welfare losses especially on agents with inelastic demand. Thus, an understanding of how price structure emerges from competition between platforms will be crucial in resolving the price issue and consumer welfare.

The study is related to an expanse of literature on two-sided markets. For instance, it is closely related in spirit with the static model of oligopoly by Katz and Shapiro (1985) and the price allocation treatment in the work of Rochet and Tirole (2003). Intuitions were equally elicited from Laffont et al, (2003) on the special case of close perfect compatibility between platforms. Analyses of markets with consumption externalities were extracted from the static model developed by Katz and Shapiro (1985). The consideration of different forms of product differentiations with varied configurations by Armstrong and Wright (2007) based on the studies by Armstrong (2006), and Cailluad and Julien (2003) was relevant only to the extent of construction of the basic model. 
This study framework consists of specific cases of the Armstrong (2006) and Caillaud and Jullien, (2003) models. In contrast with the Armstrong model, the competitive bottleneck setting was extended to allow for multi-homing on both sides of the market, a case of "global multi-homing". More precisely, we assume that all agents multi-home and that transaction fees are strictly positive. The platforms are symmetric with no product differentiations on different sides of the market. The agents, downstream mobile phone users and up-stream software producers, have a different valuation for the services of the intermediaries. While the phone user relates to the number of innovative services that can be accessed through the intermediary, the software developers' valuation depends on the size of the demand for their product. There are no exclusive contracts and agents have options of joining any platform and will only do so if transaction fee charged is lower than the expected utility. It is difficult for agents to observe their expected utilities. Transaction fee charged are fixed and independent of the performance of the intermediary.

In the spirit of Caillaud and Jullien (2002), the model construct allows for non-exclusivity, which increases the tendency for possible collusion between intermediaries to negotiate for free mutual access to platforms. While platforms simultaneously set prices, agents observe such prices and decide on which platform to a joint that will minimise cost and maximise utility. As the only medium to connect agents, intermediaries extract monopoly rent. The study allow for third degree price discrimination by intermediaries. Competing platforms set negative registration fee with minimal differential in their transaction fee (usually set at maximum). The study is restricted to the subscription for mobile applications (Mobile App) by phone users (x-type agents) from software developers (y-type agents) using multiple platforms. The above setting represent a snap shot of the Nigeria market from which basic assumptions were elicited for modelling

The major contribution of the study is the development of a theoretical model and the construction of equilibrium for the mobile communication market given the aforementioned peculiarities. In spite of the growing contribution to literature on two-sided markets, modelling of a multi-sided mobile communication market is relatively scarce especially of a developing country. While inferences from the analyses might have broader applications, they are of specific reference to the mobile communication market. Such inferences will be of practical relevance for the Nigerian Communication Commission's Consumer Affairs Bureau in policy and regulatory interventions. Following the introduction, the model was presented in section 2 while preliminary results where discuss in the last section.

\section{THE BASIC MODEL}

For analytical convenience but without loss of generality, we assume two symmetric Network Operators as platforms- MTN $(\mathrm{m})$ and Globacom $(\mathrm{g})$ that are used by two agent types: $(x-$ type and $y-$ type). The agent-types have options of joining any of the two symmetric platforms ( $\mathrm{m}$ and $\mathrm{g}$ ). We further assume that agents on both sides of the market are into multihoming. Suppose the number of x-types and y-types agents are denoted by $\left(n_{1}, n_{2}\right)$. In our twosided market, there is the existence of positive network externalities that result in the generation of surplus for both agent-types. For trade to occur, $n_{1}, n_{2}$ must interface with the existing platforms $\left(I_{m, g}\right)$. Agents are expected to act rationally in the choice of platforms and homing decision. This is needed to guarantee the feasibility of maximum surplus. In essence, we expect the decision to participate in trade to be driven by surplus generation. This exclude agents with beliefs about negative surplus from the network. Maximum value of trade is achieved for $x$-type agent only to the extent of exact matching (e. $\mathrm{g}$ an iphone owner subscribing for an iphone mobile application through the least cost platform) 
We suppose prices charged by platforms are convertible into measurable utilities obtained from services express as; $\left(U_{i}^{m}, U_{i}^{g} ; i=1,2\right)$. Given that such services are non-exclusive but homogeneous, we represent derived utilities from both sides of the market as linear functions that can be normalised to unity in the spirit of Caillaud and Jullien, (2003):

$$
U_{x}^{i}+U_{y}^{i}=1 ; i=m, g----
$$

We assume $y$-type agents to have larger expected gains based on the notion of indirect network externalities, $U_{y} \geq \frac{1}{2} \geq U_{x}$. The derived utilities, $U_{x}, U_{y}$ are conditioned on matching, with a probability $(\rho)$ that platforms will match agents types given as $(\rho \leq 1)$-indicating imperfect matching. We assume that rational agents on both sides will chose to multi-home since the matching process is not perfect $(\rho \leq 1)$ as this will enable them to connect with more agents. The efficiency of a platform is expected to increase with the probability of matching agents $(\rho)$ which is largely a function of the technology of the intermediary (the PSTN). A rational agent of any type will effect transaction only through platform that is efficient; $\rho>C_{m, g}$ where $C_{m, g}$ are costs of services charged by $I_{m, g}$ on the distinct group of agents respectively. It is expected that agents will effect transactions through platform with the minimum cost: $U_{i} \min \left\{C_{m, g},\right\}$

Suppose transaction fees $T_{m, g}$ consist of $C_{m, g}$ and a mark-up $\left(K_{i}\right)$. If intermediaries set prices simultaneously that can be observed by both agents types, agents will act correspondingly by selecting their service providers rationally to maximise their surpluses. From expression 1, the net surplus available for sharing by both agents types will be:

$S_{x, y}=1-T_{m, g} \geq 0 ; T_{m, g}=C_{i}+K_{i}------(2)$ where $S_{x, y}$ is net surplus for sharing.

While the expected utility for $x$ - type agent ;

$U_{x}^{E}=\rho U_{x}\left(S_{x}\right)--------(3)$

Two assumptions are crucial for $E q 3$ to hold: (i) agents utilities only depend on total transaction fee-from multi-homing (ii) there is no trade distortion associated with the use of transaction fees.

In this model as opposed to Caillaud and Jullien, $I_{m, g}$ set prices simultaneously with some form of cooperation, which normally ensures that $T_{m, g}>0$ on both sides of the market even with an "output tax". Such behaviour is perceived to be in violation of antitrust policy with respect to price setting under competition. It is also in contrast with the divide and conquer strategy in the Caillaud and Jullien model. In determining prices, $m$ for instance, chooses a set, $T$ and $a$, where $T$ is the transaction fee and $a$ is the access charge (cost of multi-homing though only $T$ is observable). The competing platform $g$ is guided by the margin $\left(T_{m}-a_{m}\right)$ in deciding its price system. The intermediaries operate in a market that is fully deregulated and have power to choose a retail price not limited to an average price cap constraint.

Suppose from granting access to a mobile App, $\mathrm{m}$ generates a gross utility $U$ for subscribers on both sides of the market at a cost of $C_{m}$ and charges a price $T_{m}$ per agents depending on type. The net utility per agent is $U_{m}-T_{m}$ while the rival (g) intermediary net utility $U_{g}-T_{g}$ at cost $C_{g}$ defines its maximum price $T_{g}=T_{m}-\left[U_{m}-U_{g}\right]$. From the social welfare perspective, it becomes desirable to have multiple platforms (justifying the entry of $g$ and by extension any 
other subsequent platform to enhance competition) if $C_{m} \geq C_{g}+\left[U_{m}-U_{g}\right]$ and $T_{m}-a_{m} \geq$ $C_{g}+\left[U_{m}-u_{g}\right]$.

Suppose $I_{m, g}$ incurs costs $C_{m, g}$ for $n_{1,2}$ agents on both sides, the social optimum prices are expected to satisfy $T_{m, g}=C_{m, g}-S_{x, y} n_{1,2}$. The determination of these prices are crucial from the standpoints of economic efficiency (efficient component pricing rule) and consumer welfare. However, in practice, it is unclear if the prices chosen by these intermediaries $I_{m, g}$ are flexible, efficient and based on the Ramsey principles.

Proposition 1: A market allocation exist that optimises the equilibrium outcome given the price system.

Assuming price differential between the intermediaries $\left(I_{m, g}\right)$ is negligible $P_{m}^{x} \approx P_{g}^{x}$ for a given class of $x$-type agent, it is reasonable to assert that for such a class, agents will be evenly distributed between platforms . In equilibrium, a mapping exist that relate to each price system a distribution of $x-$ type "class "agent such that:

$$
\psi_{m, g}^{x} \mid P_{m, g}=\left\{n_{i}^{m}, n_{i}^{g}\right\}
$$

where $\psi_{i}^{x}$ is the market allocation given multi-homing prices charged and $n_{i}^{m}$ is the proportion of $x-$ type of a given class registered with $m$. A mass of such agents also exist that multi-homes; $n_{i}^{H}$ (mass of agents multi-homing) such that the market allocation in equilibrium given price is $\psi_{m, g}^{x} \mid P_{m, g}=\left\{n_{i}^{m}, n_{i}^{H}, n_{i}^{g}\right\}$.

Consequently, for multi-homing to be efficient, then $\rho(1-\rho)>C_{m, g} ; n_{i}^{H}=1$. Given a define market allocation and a prevailing price system, available profit to the platforms in equilibrium will be:

$$
\pi_{m, g}\left(P_{m, g}, \psi\right)=\sum_{i=2} n_{x, y}\left(P_{x, y}-C_{x, y}\right)+\rho_{m, g} n_{x, y}---(4)
$$

$n_{x, y}$ is the market allocation that optimises $\pi_{m, g}\left(P_{m, g}, \psi\right)$ associated with $P_{m, g}$, while $\psi=$ $\left\{n_{i}^{m}, n_{i}^{g}, n_{i}^{H}\right\}$ is the distribution of $n_{i}$ amount of agents of both types across the platforms $m, g$ independently as well as those multi-homing, $H$. From (4), an equilibrium exist and consist of a system of market allocations for each possible price system. Intuitively, the price structure and the platform efficiency $\rho_{m, g} ; \rho(1-\rho)$ are relevant in the determination of total revenue $\left(n_{x, y} P_{x, y}\right)$ and equilibrium profit of the platforms.

With non-exclusivity, two optimal pricing strategies are available to $I_{m, g}$. For instance, $m$ can decide to act as a first source by charging $T_{m}<T_{g}$, thereby processing the transaction whenever there is a match among multi-homing users. Alternatively, acting as a second source $T_{m}>T_{g}$ will confer first source advantage to $g$ with $m$ only processing transaction whenever the match has failed at $g$. For a second source to be profitable, multi-homing must be efficient as the profit is bounded from above by the surplus $\rho(1-\rho)>C_{m, g}$ generated through multihoming.

Proposition 2: A multi-homing equilibrium exist in a two-sided market if $\rho(1-\rho)>C_{m, g}$ and a feasible higher equilibrium profit if there is differential in transaction fee. Similarly, a symmetrical maximal profit exist in the absence of endogenous differentiation both in services and in transaction fee. 


$$
\pi_{m}=\rho(1-\rho)+\frac{\rho^{2}(1-\rho) u_{x}}{\rho u_{y}+u_{x}}-C_{m}>\pi_{g}=\rho(1-\rho) f-C_{m, g}---(5)
$$

In the above case, $I_{m, g}$ are playing different roles with $m$ setting a lower transaction fee. The higher profit equilibrium is characterised by $T_{m, g}, \pi_{m}$, and $\pi_{g} ; T_{m}<T_{g}$. Conditions such as non-exclusivity of services and efficiency of platforms will guarantee multi-homing equilibrium. Given such conditions, a higher equilibrium profit is tenable with differential in transaction fee. We assume a two-sided effect though the surplus from y-agent is expected to be greater than that of the $x$-agents given that transaction fee charged is likely to favour $y-$ type (elasticity).

\section{Lemma 1}

Maximal symmetrical profit exist if there is no endogenous differentiation in transaction fees between platforms: $I_{m, g} ; T_{m} \approx T_{g}$ then $\pi_{m} \approx \pi_{g}=\rho(1-\rho)-C_{m, g}$. Put differently, a market sharing equilibrium exist in the presence of non-exclusivity, symmetrical price and cost structures.

To promote exclusivity, multi-homing must be discouraged. A strategy is to undercut the "fixed price", and based on monotonicity more agents will be attracted to the intermediary. Another approach is to offer cheaper exclusive contracts. If the benefit of migrating to a platform is substantially high, registration fee can be imposed and equilibrium profit increased. Intuitively, it is logical to conjecture that market performance will be enhanced with non-exclusivity accompanied by global multi-homing. Benefit of multi-homing is reduced with increased efficiency of platforms $(\rho \approx 1)$ at given costs. Negative registration fee can be use as an incentive to attract multi-homing. New migrants to the platform can access the benefits without foregoing the externalities of their previous registration.

Proposition 3: In the presence of non-exclusivity, equilibrium will fail to exist if all the intermediaries are active $\left(n_{i}^{m}>0 ; n_{i}^{g}\right)$, no multi-homing $\left(n_{x}^{H}=n_{y}^{H}=0\right)$ with $C \neq \frac{\rho}{2}$.

Without exclusivity and no multi-homing, intermediaries are constrained to charge singlehoming users an identical total price: $P_{x}^{m} \approx P_{x}^{g}$. Assuming monotonicity, an act of price-cutting will result in losses on single-homers: $P_{x}^{m} ; P_{x}^{g} \leq C_{m, g}$.

\section{PRELIMINARY RESULTS}

The model reveal some interesting results that can be taken to data for empirical evidence. From the theoretical model, non-exclusivity of services creates efficient equilibrium and allows for positive profits in equilibrium. This is largely due in part to the lessened degree of aggressive competition as compared to a situation of exclusivity. For the intermediaries (MTN and Glo), the sustenance of such equilibria depend on the efficiency of their matching technologies. This can be verify by using extreme values of the efficiency parameter and checking for the existence or otherwise of an efficient equilibrium. The efficiency of matching technique is expected to create benefits for multi homers, which must be substantial and greater than the transaction fee charged to sustain global multi-homing. The above market features seemingly generates monopoly profit with associated deadweight losses for intermediaries in the Nigerian market. A simple comparison of platform equilibria with nonexclusivity and exclusivity will be revealing.

The model also predict the feasibility of an efficient equilibrium if MTN and Glo do not induce multi-homing. When incentivise by MTN and Glo, there are strong tendencies for inefficient 
equilibrium to ensue. In other words, MTN and Glo might choose to allow for multiple registration in equilibrium.

Consumer surplus is seen to be lower with non-exclusivity. We conjecture that the bargaining process in the determination of prices is not efficient. It is also doubtful if the Baumol-Willig efficient component pricing rule (ECPR) is applied by either MTN or Glo. Transaction fee as the sole pricing instrument is used to capture efficiency gain that must have been loss by the negative registration fee. The Ramsey pricing appears to be relevant in this context as an efficient way to set prices. Such prices are used to maximise the social welfare function once a regulator specifies the measure of social welfare. However, the informational demand (information on demand elasticities) makes it less appealing. It is also strategically used for discrimination and positioning in the market. The strict enforcement of anti-trust policy will regulate any form of collusion and strengthened exclusivity, which is critical in promoting consumer welfare.

Intermediaries to obtain captive agents use negative registration fees. In addition, the possibility of price fixing on the $\mathrm{x}$-agent side of the market with a private negotiation on the $\mathrm{y}$ agent side should interest social policy makers.

\section{CONCLUSION}

The study acknowledges several theoretical and empirical tracts that are worthy of possible extensions. A significant extension among several others, is the specification of the number of agents that will join a platform resulting from the actions of intermediaries. The hotelling formula is a notable candidate. The profit functions at each strategy choice will be relevant. The sensitivity of equilibrium to price changes is equally important as well as the changes in consumer surplus.

The analysis presented above is limited to the purchase and granting of access to mobile applications conducted through platforms. As to the extent, the above model can be extended to internet access and call termination will be an interesting field for further research. In addition, bringing the model to available data so as to estimate a two-way network effects, consumer surplus and price structure among others will also be a worthy exercise.

\section{References}

Armstrong, M. (2006) “Competition in Two-Sided Markets” The RAND Journal of Economics, 37(3): 668-91

Armstrong, M. and Wright, J, (2007) Two-sided markets, competitive bottlenecks and exclusive contracts. Economic Theory 32: 353-380

Caillaud, B and Jullien, B. (2003) Chicken \& Egg: Competition among Intermediation Service Providers. RAND Journal of Economics Vol 34, issue 2, 309-28

GSMA Intelligence (2014) Analysis-Country Overview: Nigeria. Available online http://draftcontent.gsmaintelligence.com/AR/assets/4161587/GSMA_M4D_Impact_Country_Overview_Nigeria.pdf

Katz, M., L and Shapiro, C. (1985) Network Externalities, Competition, and Compatibiliy. The American Economic Review, Vol. 75 No. 3 Pp 424-440

Laffont, J.J., Rey, P., Marcus, S., and Tirole, J. (2003) “Internet Interconnection and the Off-Net-Cost Pricing Principle," Rand Journal of Economics, 34(2): 370-390

Nigeria Consumer Satisfaction Survey (NCS) Final Report Part 1 (2012). Nigeria communications commission. Available online www.ncc.gov.ng/documents/369-nigeria-consumer-satisfaction-survey-overview.

Nigerian Telecommunications Sector (NTC) summary Report (2015) National Bureau of Statistics (NBS). Available online www.nigeriastat.gov.ng/pages/download/267,2015

Rochet, J.C and Tirole, J (2003): "Platform Competition in Two-Sided Markets". Journal of the European Economic Association, 1(4): 990-1029 
Rysman, M. (2009) “The Economics of Two-Sided Markets. Journal of Economics Perspectives, Vol 23 (3) Pp125145

Wright, J. (2002) "Access Pricing Under Competition: An Application to Cellular Networks "Journal of Industrial Economics, Vol.50, Pp 289-316 